\title{
A molecular classification of human mesenchymal stromal cells
}

Florian Rohart, Elizabeth A Mason, Nicholas Matigian, Rowland Mosbergen, Othmar Korn, Tyrone Chen, Suzanne Butcher, Jatin Patel, Kerry Atkinson, Kiarash Khosrotehrani, Nicholas M Fisk, Kim-Anh K-A. Lê Cao, Christine A Wells

Mesenchymal Stromal Cells (MSC) are widely used for the study of mesenchymal tissue repair, and increasingly adopted for cell therapy, despite the lack of consensus on the identity of these cells. In part this is due to the lack of specificity of MSC markers.

Distinguishing MSC from other stromal cells such as fibroblasts is particularly difficult using standard analysis of surface proteins, and there is an urgent need for improved classification approaches. Transcriptome profiling is commonly used to describe and compare different cell types, however efforts to identify specific markers of rare cellular subsets may be confounded by the small sample sizes of most studies. Consequently, it is difficult to derive reproducible, and therefore useful markers. We addressed the question of MSC classification with a large integrative analysis of many public MSC datasets. We derived a sparse classifier (The Rohart MSC test) that accurately distinguished MSC from nonMSC samples with $>97 \%$ accuracy on an internal training set of 635 samples from 41 studies derived on 10 different microarray platforms. The classifier was validated on an external test set of 1291 samples from 65 studies derived on 15 different platforms, with $>95 \%$ accuracy. The genes that contribute to the MSC classifier formed a proteininteraction network that included known MSC markers. Further evidence of the relevance of this new MSC panel came from the high number of Mendelian disorders associated with mutations in more than $65 \%$ of the network. These result in mesenchymal defects, particularly impacting on skeletal growth and function. The Rohart MSC test is a simple in silico test that accurately discriminates MSC from fibroblasts, other adult stem/progenitor cell types or differentiated stromal cells. It has been implemented in the www.stemformatics.org resource, to assist researchers wishing to benchmark their own MSC datasets or data from the public domain. The code is available from the CRAN repository and all data used to generate the MSC test is available to download via the Gene Expression Omnibus or the Stemformatics resource. 


\section{A Molecular Classification of Human Mesenchymal Stromal Cells}

2 Florian Rohart ${ }^{1,2}$, Elizabeth Mason ${ }^{1}$, Nicholas Matigian ${ }^{1,2}$, Rowland Mosbergen ${ }^{1,5}$,

3 Othmar Korn ${ }^{1}$, Tyrone Chen ${ }^{1,5}$, Suzanne Butcher ${ }^{1,5}$, Jatin Patel ${ }^{3}$, Kerry Atkinson ${ }^{3}$,

4 Kiarash Khosrotehrani ${ }^{3,4}$, Nicholas M Fisk ${ }^{3,4}$, Kim-Anh Lê Cao ${ }^{2}$ and Christine A Wells $\mathbf{s}^{1,5^{*}}$

$5{ }^{1}$ Australian Institute for Bioengineering and Nanotechnology, The University of

6 Queensland, Brisbane, QLD Australia 4072

$7 \quad 2$ The University of Queensland Diamantina Institute, Translational Research Institute,

8 Woolloongabba, Brisbane QLD Australia, 4102

$9{ }^{3}$ The University of Queensland Centre for Clinical Research, Herston, Brisbane,

10 Queensland, Australia, 4029

$11{ }^{4}$ Centre for Advanced Prenatal Care, Royal Brisbane \& Women's Hospital, Herston,

12 Brisbane, Queensland, Australia, 4029

135 Department of Anatomy and Neuroscience, Faculty of Medicine, The University of

14 Melbourne, Victoria, 3010

$15{ }^{*}$ Correspondence to: Christine Wells, wells.c@unimelb.edu.au, @Mincle. 


\section{Introduction}

Adult tissues maintain the capacity to be replenished as part of the normal processes of homeostasis and repair. The adult stem cell hypothesis proposes that multipotent cells resident in tissues are the source of this cellular renewal, and expand in response to tissue injury. MSC were first isolated from bone marrow, where these occupy an important stem cell niche required for reconstitution of bone and the stromal compartments of marrow, and also play a supportive role in haematopoiesis (Friedenstein, Piatetzky-Shapiro \& Petrakova, 1966; Pittenger et al., 1999).

Subsequently adult stromal progenitors have been isolated and cultured from most organs including placenta, heart, adipose tissue and kidneys although the identity of these cells remains controversial (reviewed by (Phinney, 2012; Bianco et al., 2013)). Specifically, the question of how similar cells isolated outside the bone marrow niche are, and whether these could be considered bona fide MSC, or indeed challengingly, whether MSC isolated from different tissues share any phenotypic or molecular characteristics at all (Bianco et al., 2013). In this light various cells described as MSC (whether by name or attribution) have been reported as having quite different selfrenewal capacity, immunomodulatory properties or propensity to differentiate in vivo (Reinisch et al., 2014). It has been variously argued that MSC isolated from most stromal tissues are derived from perivascular progenitors (Crisan et al., 2008), or recruited from the bone marrow to distal tissue sites (Lee et al., 2010), or that resident stromal progenitors from different tissues must have tissue-restricted phenotypes. The most stringent criterion for MSC are in-vivo, bone forming capacity, however this 
38 functional standard is rarely addressed in the majority of MSC studies reported in the

39 literature to date (see for example (Reinisch et al., 2014; Sworder et al., 2015)).

40

41 Several groups have attempted to address the demand for improved molecular

42 markers, for example using global proteomics methods (Li et al., 2009), epigenetic

43 markers (de Almeida et al., 2016), transcriptome analysis of cells capable of

44 regenerating the bone marrow niche (Charbord et al., 2015), or comparison of desirable

45 properties such as capacity to form bone (Sworder et al., 2015) and indeed the studies

46 reporting global 'omic analysis of MSC number in the hundreds. Each of these studies

47 identifies a different set of potential markers, but there is little consensus among them.

48 Most human studies have been conducted on very small numbers of donors, so it is

49 difficult to dissect donor-donor heterogeneity from source heterogeneity. Nevertheless,

50 variation between MSC lines is a major contributor to differences in MSC growth and

51 differentiation capacity, and clonal variation is evident even when derived from the same

52 donor bone marrow (Samsonraj et al., 2015; Sworder et al., 2015). MSC heterogeneity

53 is further compounded by growth conditions, including the density of culture, the

54 inclusion of serum, or the substrate on which they are grown (Liu et al., 2015).

55 Consequently there is little agreement in the literature on definitive molecular or cellular 56 phenotypes of human cultured MSC, whether from bone marrow or other sources.

58 There is little consensus on whether MSC from differing tissue sources share common

59 functional attributes. The lack of definitive markers for human MSC is a major barrier to

60 understanding genuine similarities, or resolving differences between various cell 
61 sources or subsets. Even if acknowledging that there should be functional differences

62 between MSC isolated from different tissues, or donor groups, it is not clear whether

there should be any over-arching commonalities that might indicate shared homeostatic

roles or ontogenies. The field requires improved methods for benchmarking MSC

cultures, including molecular methods that lack the ambiguity of current antibody-based methods. Here we describe a sophisticated integrative transcriptome analysis of public assessment of the identity of an MSC culture.

\section{Material and Methods}

\section{Design of test and training datasets}

A careful screening of all the datasets collated in www.stemformatics.org (Wells et al.,

72 2012), GEO (Barrett et al., 2011) and ArrayExpress (Parkinson et al., 2011) at the time of this analysis identified 120 possible MSC microarray datasets. These were evaluated for the availability of the primary (unprocessed) data; unambiguous replication (biological not technical); the quality control metrics of RNA quality (5'-3' probe ratios); linear range (box-whisker plots of sample median, min and max absolute and normalized values); unambiguous sample descriptions; and sample clustering concordant with the original publication. $35 / 120$ datasets failed these criteria and were excluded from the study.

As the range of phenotypes employed across the remaining 85 MSC microarray studies was broad (Table S2), we assigned to the training group only those MSC datasets that met at least the following criteria in common: Adherence, Cell surface markers CD105+, 
$83 \mathrm{CD} 73+, \mathrm{CD} 45-$ and differentiation to at least 2 of the three MSC-definitive lineages

84 (bone, cartilage or fat), and all training datasets included substantial phenotyping above

85

86

87

these minimal criteria. These minimal common criteria were hard-coded into the Stemformatics annotation pipeline, we had a dedicated annotator responsible for the quality of these annotations and these were reviewed independently by two additional annotators. Sixteen MSC datasets met our 'gold standard' training set criteria for accompanying phenotype of MSCs, together with 27 datasets containing cells from nonmesenchymal or non-stromal sources, which we refer to as non-MSCs. In total, 41 datasets were included in the training set, with two datasets containing both MSCs and non-MSCs, with a total of 125 MSC samples and 510 non-MSC samples from 10 different microarray platforms (Table S3, accompanies the MSC clustering in Figure 2). The remaining MSC datasets were assigned to the independent test set and were used only for evaluation of accuracy of the final signature.

Details on the samples, datasets and references of the experiments can be found in Tables S2, S3 and S5. Two large datasets - 5003 (211 non-MSCs) and 6063 (45 MSCs), were subsampled prior to assigning to the training set to avoid unbalanced results. The samples left out were included in the test set (Table S5). It consisted of 65 experiments (1291 samples, 213 MSCs and 499 non-MSC) profiled across 15 different platforms.

\section{Pre-processing of data}

All data were processed using the R programming language v2.15.3 (Venables \& Smith, 2008; R Development Core Team, 2011). The pre-processing step involved a 
105 background correction performed with affy 1.36.1 and the affycoretools

106 1.30.0,gcrma 2.30.0, limma 3.14.4, lumi 2.10.0, simpleaffy 2.34.0

107 (Gautier et al., 2004, Du, Kibbe \& Lin, 2008) packages for processing of microarray data 108 depending on the platform.

109

110 Specifically, Affy GeneChips were background corrected using code:

111 data.bgonly $<-$

$112 \mathrm{bg} \cdot a d j u s t . g c r m a(d a t a$, affinity.info=affinity_data, fast=FALSE)

113 \#\# Extract GC-RMA bg-corrected expression values without re-

114 running additional bg-correction

115 data.bgexpr <- rma (data.bgonly, background=FALSE,

116 normalize=FALSE)

117 where:

118 "data" is loaded raw CEL data

119 "affinity_data" is precomputed probe affinity produced by

120 "compute.affinities()"

121

122 Affymetrix Gene ST arrays were RMA background corrected using Affymetrix

123 Power Tools v1.14.4.1 ("apt_probeset_summarize" tool). Exon probe 124 expressions were summarised to the transcript level.

125

126 Illumina chips were background corrected using code:

$127 \operatorname{lumiB}($ data, method $=\mathrm{c}($ 'bgAdjust.affy')) 
128 where:

129 "data" is non-normalized BeadStudio / GenomeStudio expression data returned by 130 "lumiR ()"

131

132 Agilent chips were background corrected using code:

133 dat <- backgroundCorrect (datraw, method="normexp",

134 normexp.method="rma" )

135 datbg <- dat [ dat\$genes\$ControlType==0, ]

136 bgave <- avereps (datbg, ID=datbg\$genes [,"ProbeName"])

138 where:

"datraw" is non-normalised Agilent data returned by "read.maimages ( ) "

All data was subsequently log2 transformed and a YuGene transformation was applied (Lê Cao et al., 2014). YuGene is a rescaling method using the cumulative proportion that is applied per sample rather than per dataset or per series. This is highly advantageous as we performed 10-fold cross-validation that would otherwise require renormalization as datasets were added or removed.

In order to combine all the datasets described in Table S2, probes were mapped to Ensembl gene to provide a common set of identifiers. Mapping thresholds of $98 \%$ match were used to align microarray probes to Ensembl human v69 transcript model cDNA and ncRNA sequences obtained from Ensembl. Transcript IDs in resulting mapping 151 were converted to Gene IDs using EnsemblBiomart v69 (Zhang et al., 2011). In the 
152 case of multi-mapping (several probes mapping to the same Ensembl gene ID), the

153 probe with the highest average expression was chosen, on a per-dataset basis.

154 The combined training data set included the gene expression measurement of 41,185

155 genes mapped by at least one probe in one dataset. When a dataset had no probes

156 mapping to a particular gene, the expression values of the gene were arbitrarily set to

157 zero for all samples from that dataset. A pre-screening step was then performed to

158 discard genes that were not present in at least half of the samples.

159 Identification of the 16-gene signature and assignation of a test sample to the MSC or non-MSC class

161 The MSC signature was identified using a novel implementation of the sparse variant of

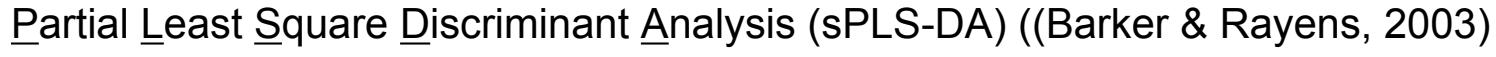
implemented for multiple microarray studies using the mixOmics package (Lê Cao et al., 2009; Lê Cao, Boitard \& Philippe, 2011). Full details of the statistical model are provided in the Supplementary methods. The underlying code for the statistical test is available as BootsPLS in the CRAN repository, and we have also made available the d3 code for

167 the interactive MSC graph implemented in Stemformatics via the BioJS framework at http://biojs.io/d/biojs-vis-rohart-msc-test

\section{Network analysis}

171 Twenty-six genes selected on component 1 equated to 20 proteins with a curated

172 interaction in the Networkanalyst protein interaction database (which draws on the PPI

173 database of the International Molecular Exchange (IMEx) consortium, accessed July

1742015 (Orchard et al., 2012; Xia, Benner \& Hancock, 2014). These seed proteins were 
175 annotated to a shortest-path first-order network of 36 nodes (16 seeds) and 48 PPI

176 edges. Twenty randomised sets of equivalent size were selected from the background

177 (expressed) genes to demonstrate a lack of PPI structure by chance. Gene ontology

178 analysis was assessed using hypergeometric mean against the Jan 2015 EBI UniProt

179 GO library (Huntley et al., 2015) Disease annotations were undertaken using the OMIM

180 (Baxevanis, 2012) and MGI (Shaw, 2009) databases. Subcellular location annotations

181 were taken from UniProt (EMBL, SIB Swiss Institute of Bioinformatics \& Protein

182 Information Resource (PIR), 2013).

183

Differential expression analysis:

185 Individual MSC markers were assessed for differential analysis between MSC and non186 MSC groups using a standard 2-tailed t-test, with a significance threshold of $10^{-6}$. For 187 exploration of MSC subsets, a linear mixed model with dataset as random effect was 188 fitted for each gene for which both the mean of bone marrow samples and other sites were higher than the median of all gene expression values. This retained 16,903 genes.

P-values were obtained by ANOVA and corrected for multiple testing with the Benjamini-Hochberg procedure (Benjamini \& Hochberg, 1995).

\section{Results}

Common MSC markers group MSC from bone marrow and other tissues.

195 The International Society for Cellular Therapy (Dominici et al., 2006) has collated a 196 large set of markers commonly used to immunophenotype MSC. These were used, in 197 combination with more recently identified markers from the current literature (Lv et al., 
198 2014), to assess whether a transcript-based approach might provide a useful molecular

199 tool to identify MSC populations (Supplementary Table S1). In order to compare data

200 generated on different microarray platforms, we built a PLS-DA matrix using these

201 markers and their corresponding expression in highly verified MSC samples. The

202 resulting scatter plot (PLS-DA, Figure 1A) demonstrated the capacity to distinguish

203 between most MSC and non-MSC samples at a transcriptional level, and further

204 showed that MSC isolated from different tissues do cluster together using these

205 markers. Figure 1B shows the 16 of 32 commonly used MSC markers that were

206 significantly differentially expressed between MSC and non-MSC groups $\left(P<10^{-6}\right)$, and

207 these included CD73 (NTE5), CD105 (Endoglin), PDGFRB and VCAM1. The average

208 expression of the remaining markers is provided in Supplementary Figure 1. Despite

209 ISCT recommendations, most of the MSC publications reviewed herein used a small

210 subset of these antibodies when phenotyping MSC, and CD73+, CD105+ and CD45-

211 were the most consistent subset used (in combination with additional markers and

212 phenotypic information, Supplementary Table 2). When just these three markers were

213 used to cluster all of the samples, $85 \%$ of MSC still grouped together $(12 / 125$

214 misclassified, Table 1, Figure 1A), but almost $12 \%$ of non-MSC samples also clustered

215 with this group. The overall accuracy increased to $92 \%$ when all 32 markers were used,

216 but the rate of non-MSC misclassification remained high $(7 \%, 35 / 510)$ and the majority

217 of these $(73.5 \%)$ were fibroblasts. It may be that these markers are less stably detected

218 at a mRNA than protein level, however this high misclassification rate is also consistent

219 with a large body of literature documenting the ambiguity of these markers, which are

220 shared with stromal fibroblasts, endothelial progenitors and hematopoietic cells. The 
221 variable expression of all 32 markers (Figure 1B, Supplementary Figure S1) is

222 consistent with the reported variability of marker use in the wider MSC research

223 community (reviewed by (Lv et al., 2014; Samsonraj et al., 2015)). Nevertheless, the

224 capacity of these known markers to cluster MSC from different studies gave us

225 confidence that a transcriptome approach was a useful and simplified alternate to

226 antibody-based protocols, so we next took an unbiased approach to find a set of

227 markers that could improve on the current classification paradigm. Our goal was to find

228 an in silico marker set that reproducibly identified bona fide MSC samples regardless of

229 platform or laboratory differences, and provide a molecular test that was simpler, and

230 more accurate than current methods.

231

232

233

234

235

236

237

238

239

240

241

242

243 244 (Supplementary Tables S2, S3).

\section{Derivation of an improved, simple and accurate in silico MSC classifier.}

A careful review of the public databases identified 120 potential MSC transcriptome studies, each comprising of a small number of donors. These were carefully curated for source, phenotypic information and growth conditions (see methods for details). From these efforts, a gold standard 'training set' was identified as meeting high confidence MSC phenotype including at least the minimal common set of CD73+, CD105+, CD45and bilineage differentiation. The training set consisted of 125 MSC samples from 16 independently derived datasets derived predominantly from bone marrow, but also included studies from other adult, neonatal and fetal stromal sources. MSC were compared to 510 definitively non-MSC samples from primary human tissues and cell lines, including cultured fibroblasts, haematopoietic cells and pluripotent stem cell lines 
245 To fully integrate and interrogate these data, we derived a novel cross-study analysis

246 framework. Our approach, described in Figure 2A, included a cross-platform

247 normalisation step (Lê Cao et al., 2014), and a modified variable (gene) selection

248 methodology. The first part of the protocol identified hundreds of potential MSC

249 markers, which in combination greatly improved the classification accuracy of $97.7 \%$

250 (Table 1). This included many of the known MSC markers. Each gene was further

251 evaluated for stability by subsampling the datasets to ensure that its inclusion was not

252 reliant on one dominant source or platform. Stability is indicated by the probability of

253 selection over 200 iterations in Figure $2 \mathrm{~B}$, and was the step that excluded most of the

254 commonly used MSC markers. For example, PDGFRB and VCAM1 were identified as

255 potential component 1 genes but their inclusion was highly variable ( 0.76 and 0.59

256 probability of selection respectively).

257

258 We reasoned that if the majority of genes discriminating between MSC and non-MSC

259 are describing a common biology and are highly correlated, then a subset of these

260 genes could be identified that would represent the entire network. Therefore we

261 iteratively assessed how the inclusion of each gene contributed to the overall accuracy

262 of the signature. This found the subset of variables that were most stable and least

263 redundant at a statistical level, and that would represent the greater network of MSC-

264 related measurements (Figure 2C). Sixteen genes were identified, collectively forming a

265 'signature', which provided a high degree of discrimination between MSC and non-MSC

266 cell types, without any loss of accuracy in accurately identifying MSC ( $>95 \%$ correct

267 MSC call or 4/125 misclassified MSC samples, Table 1) and with improved 
268 discrimination from fibroblasts and other non-MSC cell types (1.61\% false positive,

269 Table 1). We confirmed that this clustering was agnostic to technology platform or

270 manufacturer (Supplementary Figure S2).

271

272 Cells derived from bone marrow were reliably grouped together with this method (Figure

273 2D, Supplementary Figure S2E), and MSC from other tissue sources, including adipose

274 tissue, skin, lung, placenta and cord blood shared this signature. Each gene in the

275 signature made an additive contribution across 4 vectors (components), such that the

276 absolute expression of any one gene might differ from sample to sample but the

277 combination of gene expression was highly predictive. High expression of component 1

278 genes was most likely to be a positive predictor of an MSC classification (Figure 2 and

279 Supplementary Figure S3A), as indicated by the correlation of expression of each gene

280 with its component. Note that the components are linear vectors, and so a negative

281 correlation (as for component 1 genes) simply indicates the contribution of the genes to

282 clustering MSC on the positive or negative region of that component. The inclusion of

283 components 2-4 provided higher discrimination for subsets of MSC and non-MSC,

284 particularly differentiating MSC and fibroblasts derived from various tissues. These latter

285 components included stress-related genes (heat shock proteins) and early indicators of

286 lineage commitment (osteomodulin). Importantly, this multicomponent based approach,

287 in contrast to a typical differential expression analysis, allowed for a common MSC

288 phenotype that is also permissive of tissue-specific differences in the wider MSC gene

289 network. 
291 The implementation in www.stemformatics.org assessed the MSC score across 200

292 iterative predictions, where a sample must have a $95 \%$ pass rate to be classed as an

293 MSC. The distribution of the training sample scores was used to determine high

294 confidence scores (Figure 2E). By using 200 subsamplings of the training set, 200

295 scores were recorded for each sample, which enabled us to derive an individual 95\%

296 Confidence Interval ( $\mathrm{Cl})$. A sample was assigned to the MSC class if the lower bound of

297 its $95 \% \mathrm{Cl}$ is strictly higher than 0.5169 . Similarly, a non-MSC classification is given if

298 the upper bound of the $95 \% \mathrm{Cl}$ was lower than 0.4337 . Samples failing to meet these

299 criteria were assigned to an 'unknown' category. Accordingly, the four misclassified

300 MSC in the training set included one adult bone marrow MSC sample (predicted 1/200

301 times as MSC), and the remaining from two fetal studies, the first consisting of 10-week

302 chorionic villi (predicted 29/200 times as MSC) and 12-week chorionic membrane

303 preparation (2/200 MSC predictions), the second from a neonatal lung aspirate (0/200

304 positive MSC predictions).

The MSC signature genes form a cohesive network implicated in healthy

mesenchymal development and function.

309 curated set of protein-protein interactions from the BioGrid database using the genes selected from component 1 that showed a high discriminating power between MSC and

311 non-MSC. These formed a network of 36 interacting proteins (Figure $3 \mathrm{~A}$ ). The higher

312 expression of these genes in MSC samples is confirmed in Fig 3B. If the statistical tool

313 had identified a random set of genes, then the network would have little connectivity and

314 there would be no relevant functional annotations. This was confirmed by random 
315 subsampling from the background datasets, which failed to form any PPI network. To

316 assess whether the highly connected MSC network also shared any cohesive functional

317 annotations, we examined mutation databases for evidence of human diseases

318 associated with network members. A high proportion of the MSC network (30/43) are

319 represented in Mendelian disorders of mesenchymal development by virtue of their

320 mutation spectrum in facial or musculo-skeletal dysmorphologies in man, or evidence of

321 mesodermal defects in KO mouse models (Described in detail in Supplementary Table

322 S4). These included the paired-related homeobox-1 (PRRX1), a transcription factor

323 important for early embryonic skeletal and facial development, and with a de novo

324 mutation spectrum in the embryonic dysmorphology syndrome Agnathia-otocephaly

325 (Çelik et al., 2012). Likewise, mutations in bone morphogenetic protein 14

326 (BMP14/GDF5) lead to developmental abnormalities in chondrogenesis and skeletal

327 bone (Degenkolbe et al., 2013). Mutations in DDR2 cause limb defects, including

328 spondylo-epiphyseal-metaphyseal dysplasia (Ali et al., 2010) and mice over-expressing

329 DDR2 have increased body size and atypical body fat (Kawai et al., 2014). In humans,

330 Polymorphisms in $A B I 3 B P$ are associated with increased risk of osteochondropathy

331 (Zhang et al., 2014), and mice lacking Abi3bp have profound defects in MSC

332 differentiation to bone and fat (Hodgkinson et al., 2013).

333

334 We next examined functions that had been specifically validated in MSC biology,

335 specifically, whether any members of the signature had been used to prospectively

336 isolate MSC from tissue sources. ITGA11 was a member of the core signature that has

337 been used to prospectively enrich MSC from bone marrow with enhanced colony 
338 forming capacity (Kaltz et al., 2010), and independently shown to be enriched more than

3393 fold at protein level in bone marrow MSC compared to dermal fibroblasts or

340 perivascular cells (Holley et al., 2015). Although several of the known and commonly

341 used MSC markers were indeed captured in the large initial set of potential classifiers,

342 but rejected by our statistical method on the grounds of poor selection stability, these

343 were 'rescued' in the protein interaction network. That is, the behavior of these markers

344 was variable across laboratories and between microarray platforms, and often high

345 expressed on non-MSC cell types. Nevertheless, the interaction network demonstrated

346 some cohesive biology with these known markers. The most highly connected member

347 of the extended network was VCAM1, which was identified in the large prospective

348 marker set but with a low frequency of selection (0.6 on component 1 ), which eliminated

349 it from the final classifier. VCAM1, together with STRO-1, has been used for the

350 prospective isolation of human bone marrow MSC (Gronthos, 2003). VCAM1 is an

351 adhesion molecule that is induced by inflammatory stimuli to regulate leukocyte

352 adhesion to the endothelium (Dansky et al., 2001); however, in cardiac precursors its

353 expression demarcates commitment to mesenchymal rather than endothelial lineages

354 (Skelton et al., 2014).

355 Other members of our network that have been previously described in human or mouse

356 MSC biology, and used to prospectively isolate cells or have been validated at the

357 protein level include PDGFR $\beta$ (Koide et al., 2007), SPINT2 (Roversi et al., 2014),

358 CCDC80 (Charbord et al., 2015), FAP ((Bae et al., 2008), BGN (Holley et al., 2015),

359 and TM4SF1 (Bae et al., 2011). SPINT2 is a serine protease inhibitor whose activity is

360 required in bone-marrow MSC, and its loss alters hematopoietic stem cell function in 
361 myelo-dysplastic disorders (Roversi et al., 2014). In mouse, CCDC80 is also necessary

362 for reconstitution of bone marrow and support of haematopoiesis (Charbord et al., 363 2015).

364

365 The network included a high proportion of extracellular proteins $(54 \%)$ with

366 demonstrated roles in the modification of extracellular matrix proteins including

367 proteoglycans, as well as regulators of growth factor and cytokine signalling. This

368 included the cell migration inducing protein (KIAA1199/ CEMIP), which is secreted in its

369 mature form. It regulates Wnt and TGF $\beta 3$ signalling by depolarising hyaluronan, and

370 may alter trafficking of cytokines and growth factors to the extracellular milieu (Yoshida

371 et al., 2014). DDR2 is a receptor tyrosine kinase that interacts directly with collagens. It

372 stabilises the transcription factor SNAIL, and has been implicated in epithelial-

373 mesenchyme transitions in epithelial cancers (Zhang et al., 2013). CCDC80 binds

374 syndecan-heparin sulphate containing proteoglycans, has been shown to inhibit

375 WNT/beta-catenin signalling and has a regulatory role in adipogenesis (Tremblay et al.,

376 2009; Walczak et al., 2014). SRPX2 is a secreted chondroitin sulfate proteoglycan

377 involved in endothelial cell migration, tissue remodelling and vascular sprouting (Royer-

378 Zemmour et al., 2008). The chaperonins HSPB5/CRYAB and HSPB6 stabilise protein

379 complexes, and may assist in delivery of growth factor complexes where these are

380 present in high concentrations. In transplantation paradigms it is likely that the

381 therapeutic benefit derived from MSC is via local immunomodulatory, anti-inflammatory,

382 and/or trophic effects during the acute phase of cell therapy. The network of genes

383 identified here as enriched in MSC suggests an over-arching role for these cells in 
384 modifying the extracellular environment, functions important in development as well as

385 in homeostatic regulation of adult tissues.

386

387

\section{MSC differentiation, dedifferentiation and the MSC signature}

388

The majority of public microarray datasets available to us had limited phenotypic data

available, so these were not used to derive our MSC signature. Nevertheless we

390

annotated each of these samples as presumptive MSC (213 samples) or presumptive

391

non-MSC (499 samples) based on their origin and use in the source publication

392

(Supplementary Table S5). Where MSC were profiled during in vitro lineage

393

differentiation, we assigned the samples taken at intermediate time points to an

394

'unknown' category (579 samples) prior to testing these with the signature.

395

Implementation of the Rohart Test in the www.stemformatics.org resource allowed us to

evaluate a wide range of different experimental paradigms. Despite the lack of

397

phenotypic information associated with these datasets, the agreement between

398

publication status and our classification was high. Five percent of the presumptive non-

MSC (27/499) were misclassified by the signature as MSC, and around half of these

400

(>13) were neonatal or fetal dermal fibroblasts (Supplementary Table S5. Others have

401

reported MSC fractions derived from dermal tissues (reviewed in (Vaculik et al., 2012))

402

and certainly fibroblasts from other sources were not classified as MSC. Furthermore,

403

the signature could discriminate between MSC and differentiating cultures. Figure $3 \mathrm{C}$

404 demonstrates loss of the MSC score during chondrogenic differentiation with the addition of TGF $\beta$ (Dataset 6119 (Mrugala et al., 2009)) and this pattern was

406 recapitulated for cells differentiating to mineralising bone (Data not shown, but the 
407 reader is referred to the Stemformatics resource, see:

408 https://www.stemformatics.org/workbench/rohart_msc_graph?ds_id=6206\#) or to

409 adipose-like cells

410 (https://www.stemformatics.org/workbench/rohart_msc_graph?ds_id=6208\#) or when

411 undergoing reprogramming of an adipose-tissue derived iPSC

412 (https://www.stemformatics.org/workbench/rohart_msc_graph?ds_id=5018).

413

414 Comparison of MSC and adult stem/progenitor cell types

415 The limbal cell niche hosts both limbal epithelial and stromal progenitors (Lim et al., 416 2012), and the stromal progenitors were also classified as MSC by our tool (Dataset

417 6450). Some MSC subsets are likely to be derived from a perivascular progenitor. In our

418 hands, primary skeletal-muscle mesoangioblasts thought to be a subset of perivascular 419 cells in skeletal and smooth muscle (Dataset 6265 (Tedesco et al., 2012)), defined as 420 alkaline-phosphatase ${ }^{+}$CD $146^{+}$CD31/Epcam- CD56/Ncam- with demonstrated skeletal 421 muscle differentiation, were classified as MSC (Figure 3D). In contrast, the majority of

422 cells derived from a perivascular location (and confirmed as such with tissues imaged in 423 the source publication) were not classified as MSC (Figure 3E). On examining putative 424 markers of perivascular progenitors in these samples, we could demonstrate that the 425 majority of perivascular progenitors expressed higher levels of Nestin than the majority 426 of MSC (Figure 3F). MCAM+ and MCAM- cells were apparent in both MSC and 427 pericyte groups, although a higher proportion of perivascular progenitor expressed 428 MCAM RNA. In contrast, PDGFRA was highly expressed in MSC but not informative in 429 perivascular cells, and PDGFRB was highly expressed in both populations. Others have 
430 shown that high expression of PDGFRA is associated with highly proliferative MSC

431 colonies, suggesting that its expression is associated with expansion in culture

432 (Samsonraj et al., 2015). These data are consistent with a classification hierarchy

433 determined by mouse and human lineage studies, where multipotent adult cells are

434 quiescent in a perivascular location (Crisan et al., 2008; Acar et al., 2015). Thus

435 perivascular progenitor cells with MSC differentiation capacity are defined as Rohart

436 test negative, Nestin positive in our test, and as such are distinct from a Rohart test

437 positive MSC. Cells differentiating to osteoblast, chondrocyte, adipocyte or fibroblast

438 exit the MSC state and rapidly become negative for the Rohart MSC score. Given that

439 a proportion of Rohart test positive MSC express MCAM or Nestin, the classification tool

440 may detect a phenotypic spectrum that spans the intermediates across the perivascular-

441 MSC-fibroblast hierarchy.

442

443 Tissue clustering of MSC is confounded by sex and MHC-1 haplotype.

444 The capacity to group MSC-like cells is consistent with the general assumption that

445 MSC from different tissue share some common molecular properties. Many of the

446 individual studies in this reanalysis describe tissue-specific differences in MSC

447 populations. We were not able to recapitulate any of these specific differences on the

448 integrated dataset. Nevertheless, MSC from different tissues did form subclusters

449 (Supplementary Figures S2, S3), and the majority of bone marrow MSC clustered

450 together (Figure S2E). We therefore examined more broadly the genes that were

451 significantly different between bone marrow MSC and other cell types at the whole

452 transcriptome level. This analysis confirmed the observed clustering of bone marrow 
453 derived MSC, distinguished by differential expression of 425 genes (adjusted $P<0.01$,

454 Supplementary Table S6). The genes that were most differentially expressed between

455 the different MSC sources in our combined analysis were MHC class I genes, and these

456 accounted for $>40 \%$ of the top 100 differentially expressed genes in the bone-marrow

457 comparisons (Supplementary Table S6). The HLA isotypes were generally, but not

458 exclusively, expressed at lower levels in bone marrow MSC (Hierarchical Cluster,

459 Supplementary Figure S3). Estrogen and progesterone receptors, and a network of

460 associated target genes were also significantly different between tissue sources

461 (Supplementary Table S6), and this may reflect a bias in the sex of the donors from

462 which tissue was sampled; although the sex of the donors was not available for a

463 majority of samples, some tissues (such as decidual sources) will be entirely female in

464 origin. Further molecular sub-classifications of MSC will therefore require much larger

465 studies that address specific clinical or differentiation properties of the cells, and must

466 also consider ascertainment biases that may introduce confounding variables such as

467 HLA subtypes or sex.

468

469 Discussion

470 Modern molecular classification tools are needed for the characterisation of MSC ex

471 vivo and in vivo. Antibody based methods currently rely on a subset of cell surface

472 proteins that are widely acknowledged to lack specificity, and the reliability of these

473 assays is dependant on operator expertise. Our study set out to provide an alternate

474 test that had better discrimination power than current assays, was robust and easy to

475 generate. In doing so we developed a specific gene signature that is shared by a wide- 
476 variety of MSC. The "Rohart MSC test" is an in silico tool that has been implemented as

477 a simple online test that will be useful in standardisation or improvement of current bulk

478 isolation methods. This classification tool is available in the Stemformatics.org platform,

479 together with all the primary data used in derivation of the signature. Details on

480 submitting proprietary data to the Rohart test are available on the stemformatics.org 481 site.

482

483 All together we curated more than 120 MSC-related gene expression datasets in the 484 www.stemformatics.org resource (Wells et al., 2012); the datasets can be queried here using key word, dataset ID or author, together with an implementation of the Rohart 486 MSC test.

Our approach highlights the potential robustness of biological signatures when combining data from many different sources, where experimental variables such as platform or batch can be reduced (Figure S2). The methods we used for derivation of a common MSC classifier could be applied to the meta-analysis of any cell subset or phenotype where sufficient samples can be drawn from public expression databases.

The Rohart test provides a snap shot of the current state of play in MSC biology. As an in silico test it reflects all of the ambiguities existing in current nomenclature and culture practise. We anticipate that a computational classifier will evolve as the field of MSC

497 biology evolves, and as isolation methods improve. Indeed, the question of what is an $498 \mathrm{MSC}$, and whether these are a distinct stem cell population recruited from the bone 
499 marrow, as suggested by mouse studies of fetomaternal microchimerism (Seppanen et

500 al., 2013) or from perivasculature, as suggested by immunotagging of MSC-like cells

501 from perivascular regions in human tissues (Crisan et al., 2008), or are resident

502 progenitor populations specific to each organ cannot be resolved in the current study.

503 The signature itself is dependent on the quality of the MSC used in the training set. As

504 rare adult stem/progenitor cell types were under-represented in the current test or

505 training datasets, we anticipate that functional classification of MSC subtypes will

506 improve as newer sampling methods provide the means to identify and replicate these

507 cells. To highlight this point, the signature distinguishes perivascular progenitors from

508 MSC, however resolving a perivascular progenitor signature would require substantially

509 more data on this population than is currently available in the public domain. We expect

510 that further refinements in the isolation or culture of purer MSC or more precisely

511 defined functional subsets will also result in future evolutions of this in silico signature.

512

513 In summary, we set out to systematically review the current state of play in MSC biology

514 using a meta-analysis of transcriptome studies, and in doing so were able robustly to

515 identify a general MSC phenotype that could distinguish MSC from other cell types. The

516 resulting signature could also identify points of transition as MSC underwent

517 differentiation or reprogramming studies. Furthermore, we demonstrated that, at least at

518 a gene expression level, our de novo derived signature outperformed the classification

519 accuracy of the combined set of traditional MSC cell surface markers. While a signature

520 approach such as ours is not able to resolve the ontogeny or in vivo function of MSC, it

521 does provide a tool for better benchmarking and comparison of the cells grown ex vivo, 
522 and will assist with comparison of cells derived for clinical purposes. The methods that

523 we describe here, and the resulting molecular classifier represent an important step

524 towards addressing the more intractable questions of MSC identity, ontogenic

525 relationships and function.

526 


\section{References}

Acar M., Kocherlakota KS., Murphy MM., Peyer JG., Oguro H., Inra CN., Jaiyeola C., Zhao Z., Luby-Phelps K., Morrison SJ. 2015. Deep imaging of bone marrow shows non-dividing stem cells are mainly perisinusoidal. Nature 526:126-130.

Ali BR., Xu H., Akawi NA., John A., Karuvantevida NS., Langer R., Al-Gazali L., Leitinger B. 2010. Trafficking defects and loss of ligand binding are the underlying causes of all reported DDR2 missense mutations found in SMED-SL patients. Human molecular genetics 19:2239-50. DOI: 10.1093/hmg/ddq103.

Bae S., Park CW., Son HK., Ju HK., Paik D., Jeon C-J., Koh GY., Kim J., Kim H. 2008. Fibroblast activation protein alpha identifies mesenchymal stromal cells from human bone marrow. British journal of haematology 142:827-830. DOI: 10.1111/j.13652141.2008.07241.x.

Bae S., Shim SH., Park CW., Son HK., Lee HJ., Son JY., Jeon C., Kim H. 2011. Combined omics analysis identifies transmembrane 4 L6 family member 1 as a surface protein marker specific to human mesenchymal stem cells. Stem cells and development 20:197-203. DOI: 10.1089/scd.2010.0127.

Barker M., Rayens W. 2003. Partial least squares for discrimination. Journal of Chemometrics 17:166-173. DOI: 10.1002/cem.785.

Barrett T., Troup DB., Wilhite SE., Ledoux P., Evangelista C., Kim IF., Tomashevsky M., Marshall KA., Phillippy KH., Sherman PM., Muertter RN., Holko M., Ayanbule O., Yefanov A., Soboleva A. 2011. NCBI GEO: archive for functional genomics data sets-10 years on. Nucleic Acids Res 39:D1005-D1010. DOI: 10.1093/nar/gkq1184.

Baxevanis AD. 2012. Searching online mendelian inheritance in man (OMIM) for information on genetic loci involved in human disease. Current Protocols in Bioinformatics. DOI: 10.1002/0471250953.bi0102s37.

Benjamini Y., Hochberg Y. 1995. Controlling the false discovery rate: a practical and powerful approach to multiple testing. J R Stat Soc Ser B 57:289-300. DOI: 10.2307/2346101.

Bianco P., Cao X., Frenette PS., Mao JJ., Robey PG., Simmons PJ., Wang CY. 2013. The meaning, the sense and the significance: translating the science of mesenchymal stem cells into medicine. Nat Med 19:35-42. DOI: 10.1038/nm.3028.

Carvalho BS., Irizarry RA. 2010. A framework for oligonucleotide microarray preprocessing. Bioinformatics 26:2363-2367. DOI: 10.1093/bioinformatics/btq431.

Çelik T., Simsek PO., Sozen T., Ozyuncu O., Utine GE., Talim B., Yiǧit Ş., Boduroglu K., Kamnasaran D. 2012. PRRX1 is mutated in an otocephalic newborn infant conceived by consanguineous parents. Clinical Genetics 81:294-297. DOI: 10.1111/j.13990004.2011.01730.x.

Charbord P., Pouget C., Binder H., Dumont F., Stik G., Levy P., Allain F., Marchal C., Richter J., Uzan B., Pflumio F., Letourneur F., Wirth H., Dzierzak E., Traver D., Jaffredo T., Durand C. 2015. A Systems Biology Approach for Defining the Molecular Framework of the Hematopoietic Stem Cell Niche. Cell Stem Cell 15:376-391. DOI: 10.1016/j.stem.2014.06.005.

Crisan M., Yap S., Casteilla L., Chen C-W., Corselli M., Park TS., Andriolo G., Sun B., Zheng B., Zhang L., Norotte C., Teng P-N., Traas J., Schugar R., Deasy BM., Badylak S., Bühring 
H-J., Giacobino J-P., Lazzari L., Huard J., Péault B. 2008. A Perivascular Origin for Mesenchymal Stem Cells in Multiple Human Organs. Cell Stem Cell 3:301-313. DOI: http://dx.doi.org/10.1016/j.stem.2008.07.003.

Dansky HM., Barlow CB., Lominska C., Sikes JL., Kao C., Weinsaft J., Cybulsky MI., Smith JD. 2001. Adhesion of monocytes to arterial endothelium and initiation of atherosclerosis are critically dependent on vascular cell adhesion molecule-1 gene dosage. Arteriosclerosis, thrombosis, and vascular biology 21:1662-7. DOI: 10.1161/hq1001.096625.

de Almeida DC., Ferreira MRP., Franzen J., Weidner Cl., Frobel J., Zenke M., Costa IG., Wagner W. 2016. Epigenetic Classification of Human Mesenchymal Stromal Cells. Stem Cell Reports 6:168-175. DOI: 10.1016/j.stemcr.2016.01.003.

Degenkolbe E., König J., Zimmer J., Walther M., Reißner C., Nickel J., Plöger F., Raspopovic J., Sharpe J., Dathe K., Hecht JT., Mundlos S., Doelken SC., Seemann P. 2013. A GDF5 Point Mutation Strikes Twice - Causing BDA1 and SYNS2. PLoS Genet 9:e1003846.

Dominici M., Le Blanc K., Mueller I., Slaper-Cortenbach I., Marini F., Krause D., Deans R., Keating A., Prockop D., Horwitz E. 2006. Minimal criteria for defining multipotent mesenchymal stromal cells. The International Society for Cellular Therapy position statement. Cytotherapy 8:315-317. DOI: 10.1080/14653240600855905.

Du P., Kibbe WA., Lin SM. 2008. lumi: a pipeline for processing Illumina microarray. Bioinformatics 24:1547-1548. DOI: 10.1093/bioinformatics/btn224.

EMBL., SIB Swiss Institute of Bioinformatics., Protein Information Resource (PIR). 2013. UniProt. In: Nucleic acids research. 41: D43-D47.

Friedenstein AJ., Piatetzky-Shapiro II., Petrakova K V. 1966. Osteogenesis in transplants of bone marrow cells. Journal of Embryology and Experimental Morphology $16: 381-390$.

Gautier L., Cope L., Bolstad BM., Irizarry RA. 2004. affy--analysis of Affymetrix GeneChip data at the probe level. Bioinformatics 20:307-315. DOI: 10.1093/bioinformatics/btg405.

Gronthos S. 2003. Molecular and cellular characterisation of highly purified stromal stem cells derived from human bone marrow. Journal of Cell Science 116:1827-1835. DOI: $10.1242 / j c s .00369$.

Hodgkinson CP., Naidoo V., Patti KG., Gomez JA., Schmeckpeper J., Zhang Z., Davis B., Pratt RE., Mirotsou M., Dzau VJ. 2013. Abi3bp is a multifunctional autocrine/paracrine factor that regulates mesenchymal stem cell biology. Stem Cells 31:1669-1682. DOI: 10.1002/stem.1416.

Holley RJ., Tai G., Williamson AJK., Taylor S., Cain SA., Richardson SM., Merry CLR., Whetton AD., Kielty CM., Canfield AE. 2015. Comparative Quantification of the Surfaceome of Human Multipotent Mesenchymal Progenitor Cells. Stem Cell Reports. DOI: 10.1016/j.stemcr.2015.01.007.

Huntley RP., Sawford T., Mutowo-Meullenet P., Shypitsyna A., Bonilla C., Martin MJ., O'Donovan C. 2015. The GOA database: Gene Ontology annotation updates for 2015. Nucleic Acids Research 43 :D1057-D1063. DOI: 10.1093/nar/gku1113.

Kaltz N., Ringe J., Holzwarth C., Charbord P., Niemeyer M., Jacobs VR., Peschel C., Häupl T., Oostendorp RAJ. 2010. Novel markers of mesenchymal stem cells defined by genomewide gene expression analysis of stromal cells from different sources. Experimental cell research 316:2609-17. DOI: 10.1016/j.yexcr.2010.06.002. 
614

615

616

617

618

619

620

621

622

623

624

625

626

627

628

629

630

631

632

633

634

635

636

637

638

639

640

641

642

643

644

645

646

647

648

649

650

651

652

653

654

655

656

657

Kawai I., Matsumura H., Fujii W., Naito K., Kusakabe K., Kiso Y., Kano K. 2014. Discoidin domain receptor 2 (DDR2) regulates body size and fat metabolism in mice. Transgenic Research 23:165-175. DOI: 10.1007/s11248-013-9751-2.

Koide Y., Morikawa S., Mabuchi Y., Muguruma Y., Hiratsu E., Hasegawa K., Kobayashi M., Ando K., Kinjo K., Okano H., Matsuzaki Y. 2007. Two distinct stem cell lineages in murine bone marrow. Stem Cells 25:1213-1221. DOI: 2006-0325 [pii]/r10.1634/stemcells.20060325.

Lê Cao KA., Martin P., Robert-Granié C., Besse P. 2009. Sparse canonical methods for biological data integration: application to a cross-platform study. BMC Bioinformatics 10:34.

Lê Cao K-A., Rohart F., McHugh L., Korn O., Wells CA. 2014. YuGene: A simple approach to scale gene expression data derived from different platforms for integrated analyses. Genomics 103:239-251. DOI: http://dx.doi.org/10.1016/j.ygeno.2014.03.001.

Lê Cao KA., Boitard S., Philippe B. 2011. Sparse PLS discriminant analysis: biologically relevant feature selection and graphical displays for multiclass problems. BMC Bioinformatics 12.

Lee ESM., Bou-Gharios G., Seppanen E., Khosrotehrani K., Fisk NM. 2010. Fetal stem cell microchimerism: natural-born healers or killers? Molecular Human Reproduction 16 :869_ 878. DOI: 10.1093/molehr/gaq067.

Li G., Zhang XA., Wang H., Wang X., Meng CL., Chan CY., Yew DTW., Tsang KS., Li K., Tsai SN., Ngai SM., Han ZC., Lin MCM., He ML., Kung HF. 2009. Comparative proteomic analysis of mesenchymal stem cells derived from human bone marrow, umbilical cord, and placenta: Implication in the migration. Proteomics 9:20-30.

Lim MN., Hussin NH., Othman A., Umapathy T., Baharuddin P., Jamal R., Zakaria Z. 2012. Ex vivo expanded SSEA-4+ human limbal stromal cells are multipotent and do not express other embryonic stem cell markers. Molecular vision 18:1289-300.

Liu Y., Muñoz N., Bunnell BA., Logan TM., Ma T. 2015. Density-Dependent Metabolic Heterogeneity in Human Mesenchymal Stem Cells. STEM CELLS:n/a-n/a. DOI: 10.1002/stem.2097.

Lv F-J., Tuan RS., Cheung KMC., Leung VYL. 2014. Concise review: the surface markers and identity of human mesenchymal stem cells. Stem cells (Dayton, Ohio) 32:1408-19. DOI: 10.1002/stem.1681.

Mrugala D., Dossat N., Ringe J., Delorme B., Coffy A., Bony C., Charbord P., Haupl T., Daures JP., Noel D., Jorgensen C. 2009. Gene expression profile of multipotent mesenchymal stromal cells: Identification of pathways common to TGFbeta3/BMP2-induced chondrogenesis. Cloning Stem Cells 11:61-76. DOI: 10.1089/clo.2008.0070.

Orchard S., Kerrien S., Abbani S., Aranda B., Bhate J., Bidwell S., Bridge A., Briganti L., Brinkman FSL., Cesareni G., Chatr-aryamontri A., Chautard E., Chen C., Dumousseau M., Goll J., Hancock REW., Hannick LI., Jurisica I., Khadake J., Lynn DJ., Mahadevan U., Perfetto L., Raghunath A., Ricard-Blum S., Roechert B., Salwinski L., Stumpflen V., Tyers M., Uetz P., Xenarios I., Hermjakob H. 2012. Protein interaction data curation: the International Molecular Exchange (IMEx) consortium. Nat Meth 9:345-350.

Parkinson H., Sarkans U., Kolesnikov N., Abeygunawardena N., Burdett T., Dylag M., Emam I., Farne A., Hastings E., Holloway E., Kurbatova N., Lukk M., Malone J., Mani R., Pilicheva E., Rustici G., Sharma A., Williams E., Adamusiak T., Brandizi M., Sklyar N., Brazma A. 
658

659

660

661

662

663

664

665

666

667

668

669

670

671

672

673

674

675

676

677

678

679

680

681

682

683

684

685

686

687

688

689

690

691

692

693

694

695

696

697

698

699

700

701

702

2011. ArrayExpress update-an archive of microarray and high-throughput sequencingbased functional genomics experiments. Nucleic Acids Res 39:D1002-D1004. DOI: 10.1093/nar/gkq1040.

Phinney DG. 2012. Functional heterogeneity of mesenchymal stem cells: Implications for cell therapy. J Cell Biochem 113:2806-2812. DOI: 10.1002/jcb.24166.

Pittenger MF., Mackay AM., Beck SC., Jaiswal RK., Douglas R., Mosca JD., Moorman MA., Simonetti DW., Craig S., Marshak DR. 1999. Multilineage potential of adult human mesenchymal stem cells. Science 284:143-147.

R Development Core Team R. 2011. R: A Language and Environment for Statistical Computing. $R$ Foundation for Statistical Computing 1:409. DOI: 10.1007/978-3-540-74686-7.

Reinisch A., Etchart N., Thomas D., Hofmann NA., Fruehwirth M., Sinha S., Chan CK., Senarath-Yapa K., Seo E-Y., Wearda T., Hartwig UF., Beham-Schmid C., Trajanoski S., Lin Q., Wagner W., Dullin C., Alves F., Andreeff M., Weissman IL., Longaker MT., Schallmoser K., Majeti R., Strunk D. 2014. Epigenetic and in vivo comparison of diverse MSC sources reveals an endochondral signature for human hematopoietic niche formation. Blood 125:249-260.

Roversi FM., Lopes MR., Machado-Neto JA., Longhini ALF., Duarte A da SS., Baratti MO., Palodetto B., Corrocher FA., Pericole FV., Campos P de M., Favaro P., Traina F., Saad STO. 2014. Serine protease inhibitor kunitz-type 2 is downregulated in myelodysplastic syndromes and modulates cell-cell adhesion. Stem cells and development 23:1109-20. DOI: $10.1089 / \mathrm{scd} .2013 .0441$.

Royer-Zemmour B., Ponsole-Lenfant M., Gara H., Roll P., Lévêque C., Massacrier A., Ferracci G., Cillario J., Robaglia-Schlupp A., Vincentelli R., Cau P., Szepetowski P. 2008. Epileptic and developmental disorders of the speech cortex: Ligand/ receptor interaction of wild-type and mutant SRPX2 with the plasminogen activator receptor UPAR. Human Molecular Genetics 17:3617-30. DOI: 10.1093/hmg/ddn256.

Samsonraj RM., Rai B., Sathiyanathan P., Puan KJ., Rötzschke O., Hui JH., Raghunath M., Stanton LW., Nurcombe V., Cool SM. 2015. Establishing Criteria for Human Mesenchymal Stem Cell Potency. STEM CELLS 33:1878-1891. DOI: 10.1002/stem.1982.

Seppanen E., Roy E., Ellis R., Bou-Gharios G., Fisk NM., Khosrotehrani K. 2013. Distant mesenchymal progenitors contribute to skin wound healing and produce collagen: evidence from a murine fetal microchimerism model. PLoS One 8:e62662. DOI: 10.1371/journal.pone.0062662.

Shaw DR. 2009. Searching the Mouse Genome Informatics (MGI) resources for information on mouse biology from genotype to phenotype. Current protocols in bioinformatics / editoral board, Andreas D. Baxevanis ... [et al.] Chapter 1:Unit1.7. DOI: 10.1002/0471250953.bi0107s25.

Skelton RJP., Costa M., Anderson DJ., Bruveris F., Finnin BW., Koutsis K., Arasaratnam D., White AJ., Rafii A., Ng ES., Elefanty AG., Stanley EG., Pouton CW., Haynes JM., Ardehali R., Davis RP., Mummery CL., Elliott DA. 2014. SIRPA, VCAM1 and CD34 identify discrete lineages during early human cardiovascular development. Stem Cell Research 13:172179. DOI: 10.1016/j.scr.2014.04.016.

Sworder BJ., Yoshizawa S., Mishra PJ., Cherman N., Kuznetsov SA., Merlino G., Balakumaran A., Robey PG. 2015. Molecular profile of clonal strains of human skeletal stem/progenitor cells with different potencies. Stem cell research 14:297-306. DOI: 
703

704

705

706

707

708

709

710

711

712

713

714

715

716

717

718

719

720

721

722

723

724

725

726

727

728

729

730

731

732

733

734

735

736

737

738

739

740

741

742

10.1016/j.scr.2015.02.005.

Tedesco FS., Gerli MFM., Perani L., Benedetti S., Ungaro F., Cassano M., Antonini S., Tagliafico E., Artusi V., Longa E., Tonlorenzi R., Ragazzi M., Calderazzi G., Hoshiya H., Cappellari O., Mora M., Schoser B., Schneiderat P., Oshimura M., Bottinelli R., Sampaolesi M., Torrente Y., Broccoli V., Cossu G. 2012. Transplantation of Genetically Corrected Human iPSC-Derived Progenitors in Mice with Limb-Girdle Muscular Dystrophy. Science Translational Medicine 4:140ra89. DOI: 10.1126/scitranslmed.3003541.

Tremblay F., Revett T., Huard C., Zhang Y., Tobin JF., Martinez R V., Gimeno RE. 2009. Bidirectional modulation of adipogenesis by the secreted protein Ccdc80/DRO1/URB. Journal of Biological Chemistry 284:8136-8147. DOI: 10.1074/jbc.M809535200.

Vaculik C., Schuster C., Bauer W., Iram N., Pfisterer K., Kramer G., Reinisch A., Strunk D., Elbe-Burger A. 2012. Human Dermis Harbors Distinct Mesenchymal Stromal Cell Subsets. J Invest Dermatol 132:563-574.

Venables WN., Smith DM. 2008. R Development Core Team. An Introduction to R Notes on $R$ A Programming Environment for Data Analysis and Graphics $R$ core team version 2:R: A language and environment for statistical comp.

Walczak EM., Kuick R., Finco I., Bohin N., Hrycaj SM., Wellik DM., Hammer GD. 2014. WntSignaling Inhibits Adrenal Steroidogenesis by Cell-Autonomous and Non-Cell-Autonomous Mechanisms. Molecular endocrinology (Baltimore, Md.):me20141060. DOI: 10.1210/me.2014-1060.

Wells CA., Mosbergen R., Korn O., Choi J., Seidenman N., Matigian NA., Vitale AM., Shepherd J. 2012. Stemformatics: Visualisation and sharing of stem cell gene expression. Stem Cell Research 10:387-395. DOI: http://dx.doi.org/10.1016/j.scr.2012.12.003.

Xia J., Benner MJ., Hancock REW. 2014. NetworkAnalyst - integrative approaches for proteinprotein interaction network analysis and visual exploration. Nucleic Acids Research 42 :W167-W174. DOI: 10.1093/nar/gku443.

Yoshida H., Nagaoka A., Nakamura S., Tobiishi M., Sugiyama Y., Inoue S. 2014. N-Terminal signal sequence is required for cellular trafficking and hyaluronan-depolymerization of KIAA1199. FEBS letters 588:111-6. DOI: 10.1016/j.febslet.2013.11.017.

Zhang J., Haider S., Baran J., Cros A., Guberman JM., Hsu J., Liang Y., Yao L., Kasprzyk A. 2011. BioMart: a data federation framework for large collaborative projects. Database (Oxford) 2011:bar038. DOI: bar038 [pii]10.1093/database/bar038.

Zhang K., Corsa CA., Ponik SM., Prior JL., Piwnica-Worms D., Eliceiri KW., Keely PJ., Longmore GD. 2013. The collagen receptor discoidin domain receptor 2 stabilizes SNAIL1 to facilitate breast cancer metastasis. Nature cell biology 15:677-87. DOI: 10.1038/ncb2743.

Zhang F., Guo X., Zhang Y., Wen Y., Wang W., Wang S., Yang T., Shen H., Chen X., Tian Q., Tan L., Deng H-W. 2014. Genome-wide copy number variation study and gene expression analysis identify ABI3BP as a susceptibility gene for Kashin-Beck disease. Human Genetics 133:793-799. DOI: 10.1007/s00439-014-1418-4. 


\section{Table $\mathbf{1}$ (on next page)}

Table 1

Table 1: MSC Signature improves the classification accuracy of MSC compared to a panel of $\mathbf{3 2}$ commonly used MSC markers. Column 1 provides the comparison of the classification accuracy of the 635 training samples using (Column 2) the 3 markers used as the minimal immunophenotype of the MSC training samples. (Column 3) a panel of 32 commonly used immune-markers in the MSC literature; (Column 4) using the unrefined SPLSDA output; or (Column 5) with our final signature of 16 genes. Performance of each gene group was assessed using 200 random subsamplings of the training set. The internal classification error rate was calculated from a PLS-DA with 2 components (known immunemarkers), or was an output of our statistical model with genes selected in an unbiased manner (cf Figure 1A). 
1 Table 1: MSC Signature improves the classification accuracy of MSC compared to

2 a panel of 32 commonly used MSC markers. Column 1 provides the comparison of

3 the classification accuracy of the 635 training samples using (Column 2) the 3 markers

4 used as the minimal immunophenotype of the MSC training samples. (Column 3) a

5 panel of 32 commonly used immune-markers in the MSC literature; (Column 4) using

6 the unrefined SPLS-DA output; or (Column 5) with our final signature of 16 genes.

7 Performance of each gene group was assessed using 200 random subsamplings of the

8 training set. The internal classification error rate was calculated from a PLS-DA with 2

9 components (known immune-markers), or was an output of our statistical model with

10 genes selected in an unbiased manner (cf Figure 1A).

\begin{tabular}{|c|c|c|c|c|}
\hline & $\begin{array}{l}C D 45 \\
C D 73 \\
C D 105\end{array}$ & $\begin{array}{c}32 \text { common } \\
\text { MSC } \\
\text { markers }\end{array}$ & $\begin{array}{l}\text { SPLS-DA prior } \\
\text { to stable gene } \\
\text { selection }\end{array}$ & $\begin{array}{c}\text { The 16-gene MSC } \\
\text { signature }\end{array}$ \\
\hline $\begin{array}{l}\text { Overall accuracy } \\
\text { (\% of } 635 \text { samples) }\end{array}$ & 87.86 & 92.33 & 97.71 & 97.85 \\
\hline $\begin{array}{l}\text { MSC misclassified } \\
\text { (\% of } 125 \text { samples) }\end{array}$ & 14.40 & 11.10 & 3.04 & 4.31 \\
\hline $\begin{array}{c}\text { Non-MSC } \\
\text { misclassified } \\
\text { (\% of } 510 \text { samples) }\end{array}$ & 11.60 & 6.82 & 2.11 & 1.61 \\
\hline
\end{tabular}

11 


\section{1}

Figure 1.Evaluation of Common MSC markers as transcriptional classifiers.

A) PLS-DA scatter plot of MSC (circles) and non-MSC cell types (triangles). Red symbols indicate cells which are incorrectly classified by the PLS-DA matrix. The matrix components consisted of 32 commonly used MSC markers. B) Box and Whisker plots showing average expression of common MSC markers that are significantly differentially expressed (t-test, $\left.P>10^{-6}\right)$ between MSC $(n=125)$ and non-MSC $(n=510)$ cell types. See also Figure S1 and Table S1. 
A

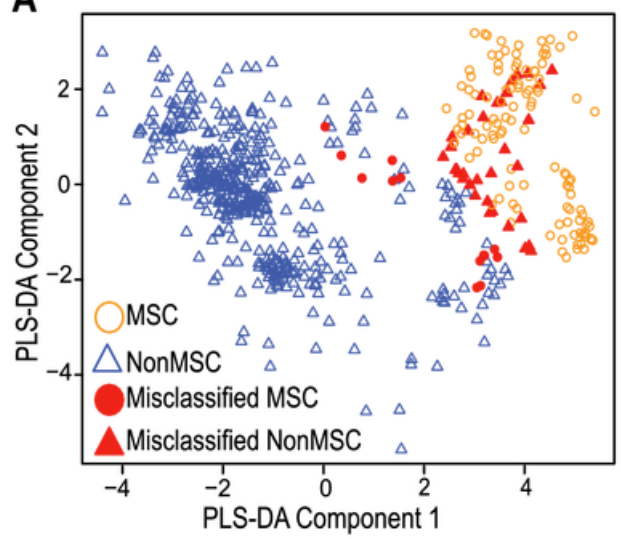

B

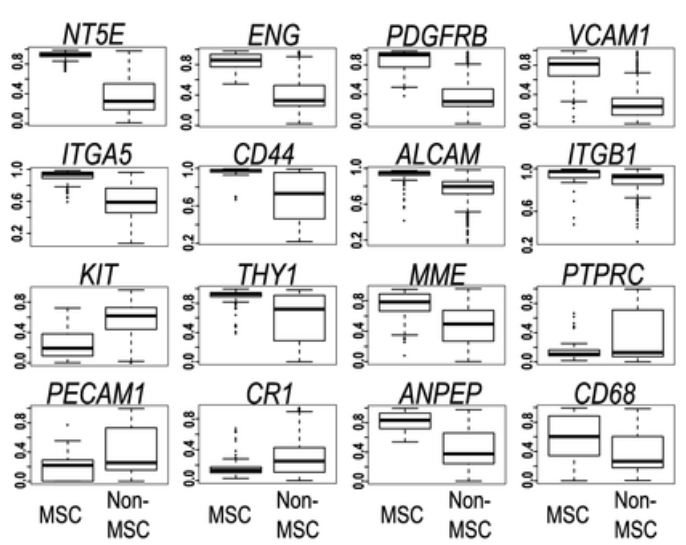


2

Figure 2.An improved in silico MSC signature.

A) Workflow summarizing the modified implementation of thesPLS-DA to integrate and evaluate cross-platform studies for derivation of a stable classifier; B) Evaluation of the stability of each gene across four components, where frequency of selection over 200 subsamplings (Y-axis) is shown per gene (ENSEMBL ID, X-axis). Labels are provided for the 16 genes contributing to the signature across 4 components. Component 1 (green), Component 2 (Blue), Component 3 (Brown), Component 4 (Black). Small text gene symbols indicate a selection of previously identified MSC markers that were excluded for poor stability. C) Evaluation of the contribution of each gene to the informativeness of its component. Each dot is a gene set, ordered along the $x$-axis by decreasing stability (frequency of selection). The $y$ axis represents the $-\log _{10}(P$-value $)$ of a one tailed $t$-test indicating the improvement in classification accuracy across 4 components. D) PLS-DA scatter plot showing sample clustering and classification accuracy of the training set (635 samples) in two components (Component $1 \mathrm{X}$ axis, Component $2 \mathrm{Y}$-axis). MSC samples are shown as circles, non-MSC as triangles, and misclassified samples are coloured red. E) Identifying the scores that classify an MSC or non-MSC. Distribution of the Rohart MSC Score (X-axis) and the distribution density (Y-axis) for samples in the MSC $(n=115)$ or non-MSC $(n=510)$ classes. Arrows indicate the scores that $99 \%$ of each class fall into. The overlap indicates the region of uncertainty, where a classification is given as 'unknown'. F) A summary of the 16-gene MSC signature colour coded to the component (as described in 1B). Gene ID is given as HUGO symbol and ENSEMBL gene ID; $\mathrm{C}$ is component; $\mathrm{P}$ is probability of selection (indicating stability); $\mathrm{R}$ is correlation of gene to component (as per 1D); $L$ is predicted subcellular location of Intracellular (I), Nucleus (N), Extracellular matrix (ECM), Secreted (S), Membrane (M) and U is unknown. See also Supplementary Figure S2 and Supplemental Tables S2, S3. 

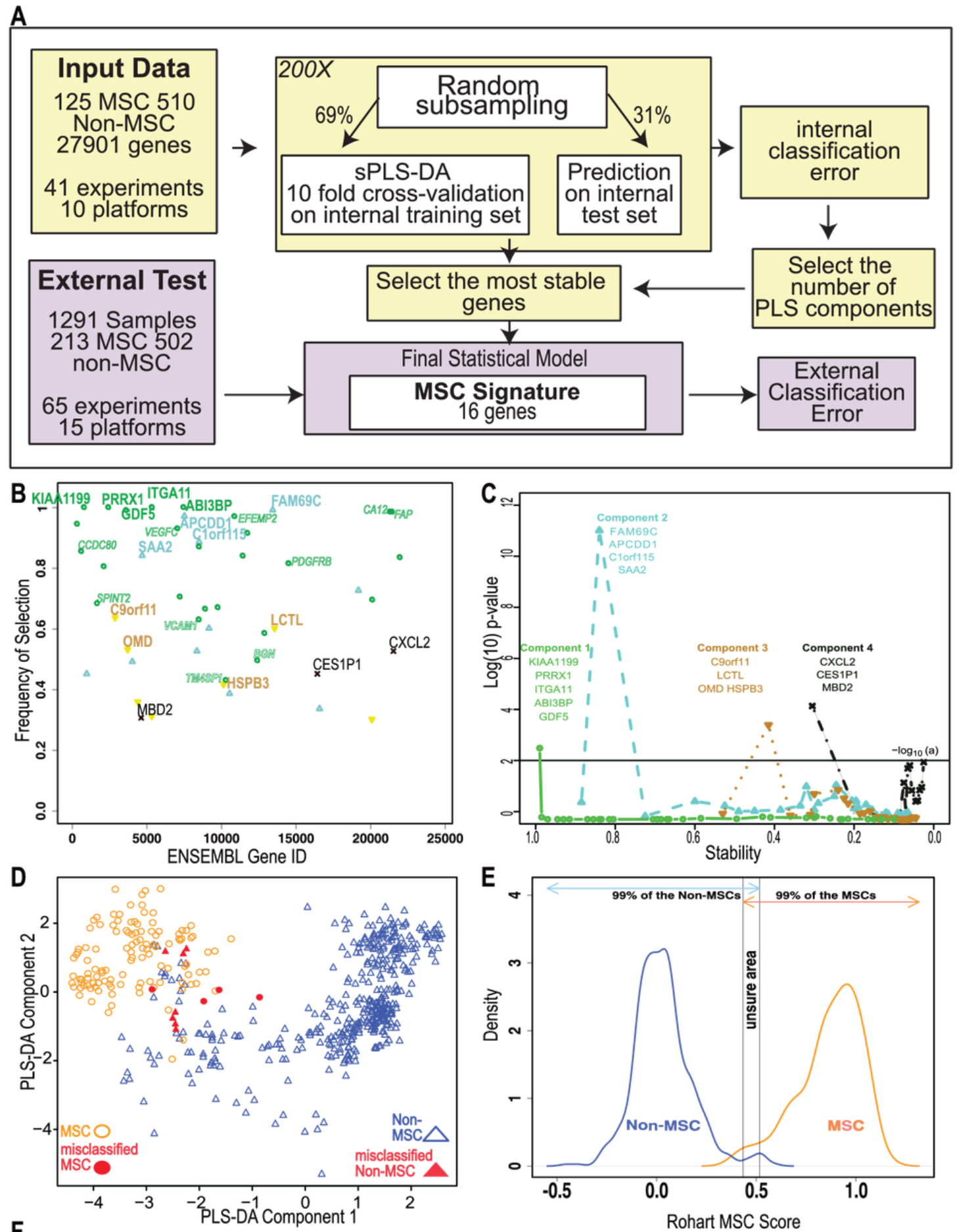

F

\begin{tabular}{|c|c|c|c|c|c|c|}
\hline $\begin{array}{l}\text { ENSEMBL } \\
\text { Gene ID }\end{array}$ & $\mathrm{C}$ & $\mathrm{P}$ & R & L & SYMBOL & Description \\
\hline ENSG00000134046 & 1 & 1 & -0.93 & ECM & KIAA1199 & Cell migration inducing protein, CEMIP \\
\hline ENSG00000116132 & 1 & 1 & -0.94 & $\mathrm{~N}$ & PRRX1 & Paired related homeobox 1 \\
\hline ENSG00000137809 & 1 & 1 & -0.83 & $\mathrm{M}$ & ITGA11 & Integrin alpha 11 , beta 1 \\
\hline ENSG00000154175 & 1 & 1 & -0.91 & ECM & $\mathrm{ABI3BP}$ & ABI family member 3 (NESH) Binding Protein, TARSH \\
\hline ENSG00000125965 & 1 & 0.99 & -0.92 & S & GDF5 & Growth differentiation factor 5, BMP14 \\
\hline ENSG00000187773 & 2 & 0.99 & -0.55 & 1 & FAM69C & Cysteine-rich type II transmembrane protein \\
\hline ENSG00000154856 & 2 & 0.96 & -0.70 & $\mathrm{M}$ & APCDD1 & Adenomatosis polyposis coli down regulated 1 \\
\hline ENSG00000162817 & $\underline{2}$ & 0.89 & -0.74 & $\mathrm{U}(\mathrm{M})$ & C1orf115 & Chromosome 1 uncharacterised open reading frame 115 \\
\hline ENSG000000134339 & 2 & 0.82 & -0.80 & $\mathrm{~S}$ & SAA2 & Serum amyloid A2 \\
\hline ENSG00000120160 & 3 & 0.66 & 0.63 & 1 & C9orf11 & Sperm acrosome associated, Equatorin \\
\hline ENSG00000188501 & 3 & 0.61 & -0.68 & T & LCTL & Lactase-like 1 \\
\hline ENSG00000127083 & 3 & 0.57 & -0.42 & ECM & $\mathrm{OMD}$ & Osteomodulin \\
\hline ENSG000000169271 & 3 & 0.41 & 0.58 & $\mathrm{~N}$ & HSPB3 & Heat shock $27 \mathrm{kDa}$ protein 3 \\
\hline ENSG0000081041 & 4 & 0.53 & 0.54 & $\mathrm{~S}$ & CXCL2 & Chemokine (C-X-C motif) ligand 2 \\
\hline EReerbocevievsin & RDF & Q. $4 \mathrm{BC}$ & 16.032 & 9002 & :1@्CRERIE & 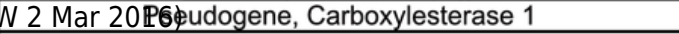 \\
\hline 00134046 & 4 & 0.31 & 0.74 & $\mathrm{~N}$ & MBD2 & Methyl-CpG binding protein 2 \\
\hline
\end{tabular}




\section{3}

Figure 3: The MSC signature forms part of a network of extracellular proteins and discriminates between differentiating or related adult stem cell types.

A) An extended protein-protein network diagram of the Rohart MSC signature genes demonstrating a role for VCAM1 and PDGFRB as part of a functionally interconnected set of glycoproteins, integrins, growth factors and extracellular matrix proteins.Green nodes are seed network members from component 1 genes, white nodes are inferred network members, and edges are protein-protein interactions. B) Box and Whisker plots showing average expression of the genes making up the MSC signature component 1 genes in MSC $(n=115)$ and non-MSC $(n=510)$. Note, PRRX1, GDF5, ITGA11 and ABI3BP also form seeds in the network. KIAA1199 lacks PPI data and is not annotated in the network. C) Classification of bone marrow MSC over a time course of differentiation to cartilage; $y$-axis gives the Rohart score, $x$-axis orders the samples from each experimental series. Three differentiation series from three donors are shown. The uncertainty region stands between the MSC and non-MSC prediction regions. D) Classification of perivascular-derived stem cells from skeletal muscle mesangioblasts (HMAB), or iPSC-derived mesangioblasts (HIDEM) from donors with muscular dystrophy (MD) or healthy donors (WT). Error bars around each prediction score represent the $\mathrm{Cl}$ boundaries. A sample is classified as 'unsure' (indicated in grey) if its prediction score or its $\mathrm{Cl}$ overlapped the uncertainty region. E) Classification of pericytes derived from three distinct datasets: from Left-Right neonatal foreskin (Antigen HD-1 dim or bright); placental pericytes; perivascular endometrial stem cells (CD146+/PDGRFB+). Stemformatics dataset identifiers provided for each experimental series. Error bars around each prediction score represent the $\mathrm{Cl}$ boundaries. F) Distribution of expression of common MSC/Pericyte markers. $\mathrm{X}$-axis is Gene expression ranked by the YuGene cumulative proportion, $\mathrm{Y}$-axis is the density distribution of MSC (orange plot, $n=115$ ) or pericytes (black plot, $n=16$ ). See also Supplemental Figure S3 and supplemental tables S4, S5 and S6. 

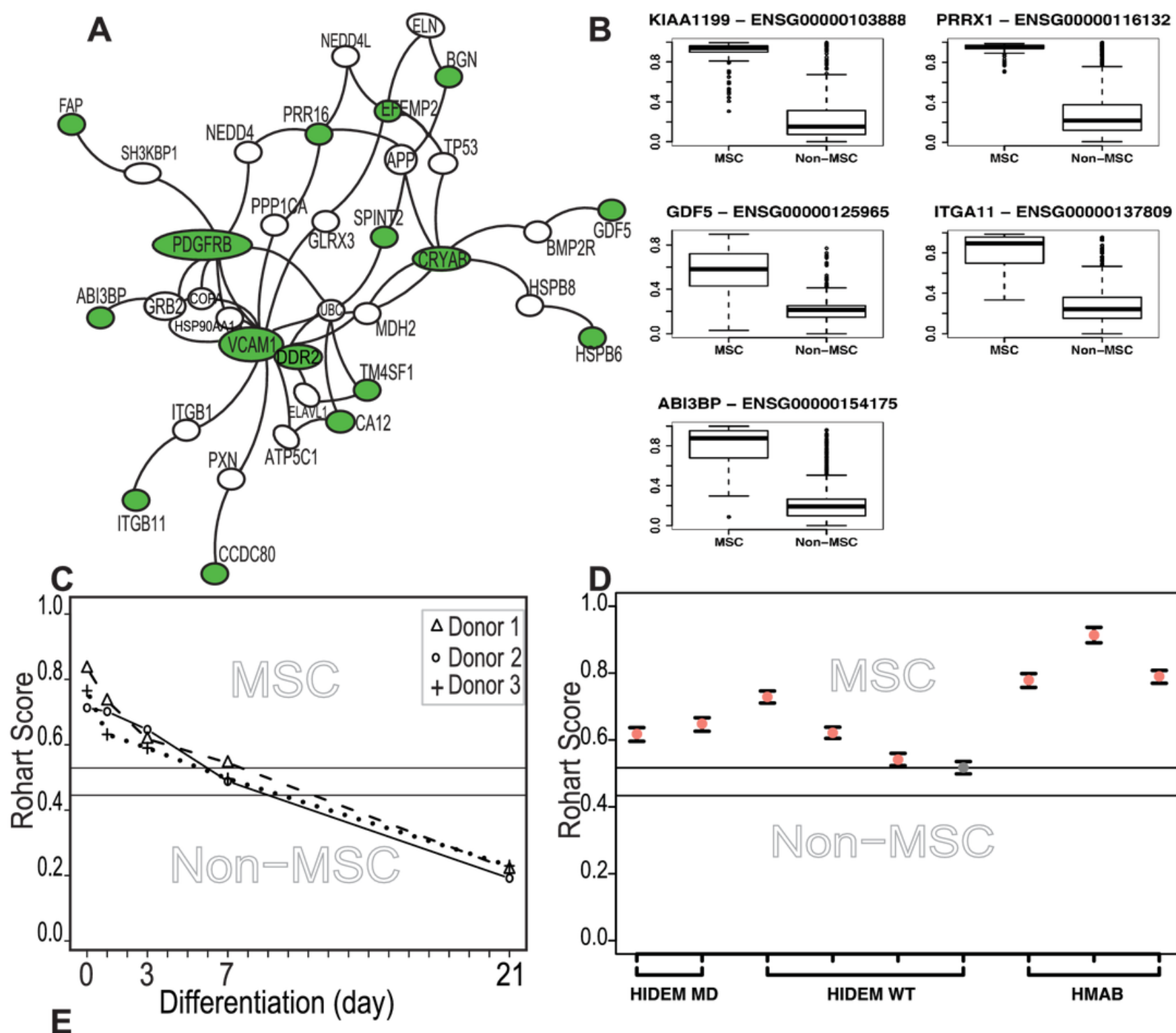

GDF5 - ENSG00000125965
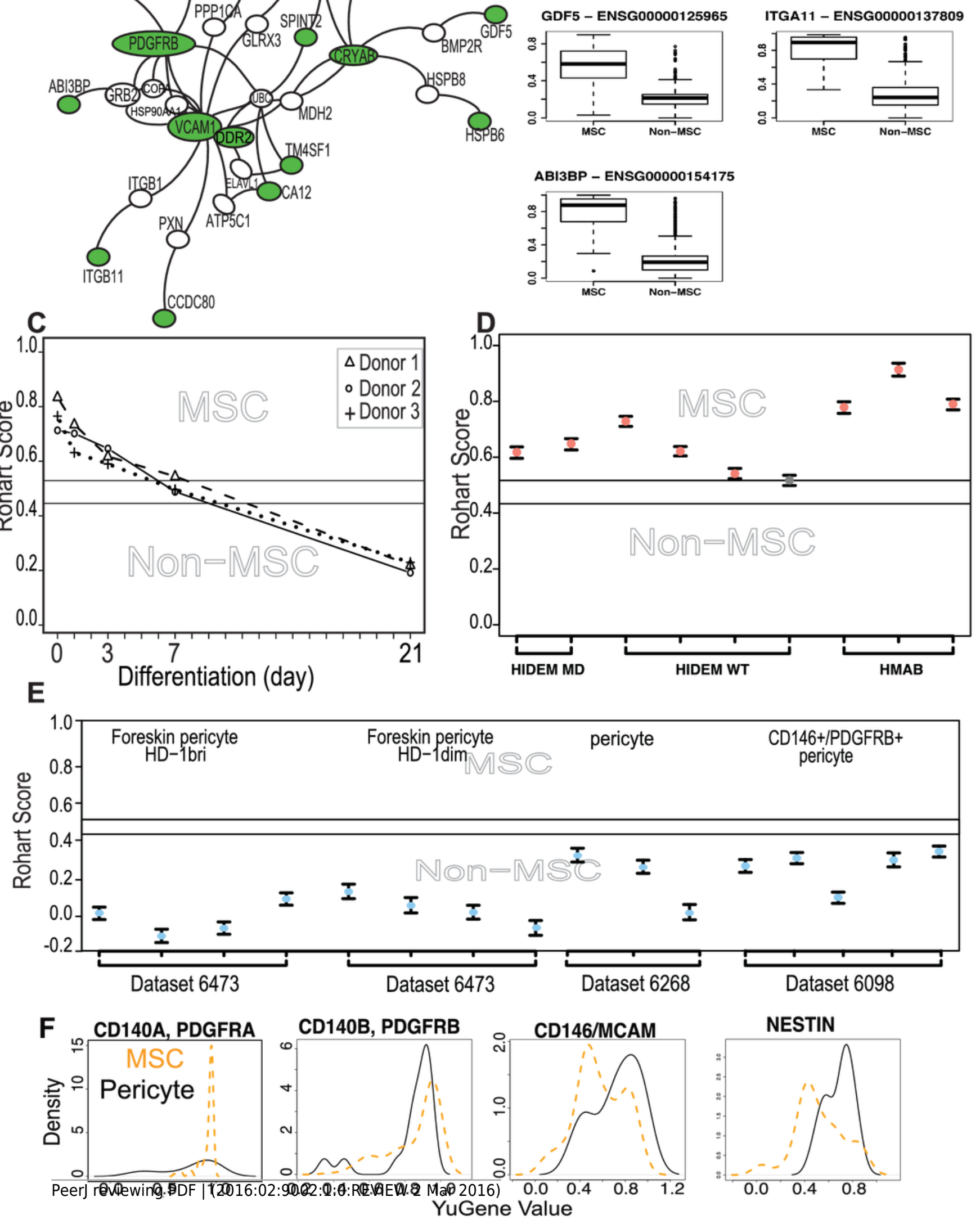